\title{
A SIMPLE PROOF OF THE $p$-ADIC VERSION OF THE SOBOLEV EMBEDDING THEOREM
}

\author{
YONG-CHEOL KIM
}

Abstract. We give a simple proof of certain mapping properties of the $p$-adic Riesz potential and Bessel potential, and the $p$-adic version of the Sobolev embedding theorem obtained in [6].

\section{Introduction}

For a prime number $p$, let $\mathbb{Q}_{p}$ denote the $p$-adic field, and let $\mathbb{Z}_{p}=\{0,1, \ldots$, $p-1\}$ and $\mathbb{Q}_{p}^{*}=\mathbb{Q}_{p} \backslash\{0\}$. By the standard $p$-adic analysis [8], we see that any non-zero element $x \in \mathbb{Q}_{p}$ is uniquely represented in the canonical form

$$
x=\sum_{j=\gamma}^{\infty} x_{j} p^{j}, \gamma=\gamma(x) \in \mathbb{Z},
$$

where $x_{j} \in \mathbb{Z}_{p}$ and $x_{\gamma} \neq 0$. Here the integer $\gamma=\gamma(x)$ is called the $p$ adic valuation of $x$ and we write $\gamma=\operatorname{ord}_{p}(x)$ with convention $\operatorname{ord}_{p}(0)=\infty$. Then it is well-known $[1,8]$ that the nonnegative function $|\cdot|_{p}$ on $\mathbb{Q}_{p}$ given by $|x|_{p}=p^{-\operatorname{ord}_{p}(x)}$ becomes a non-Archimedean norm on $\mathbb{Q}_{p}$ and $\mathbb{Q}_{p}$ is defined as the completion of $\mathbb{Q}$ with respect to the norm $|\cdot|_{p}$. For $d \in \mathbb{N}$, let $\mathbb{Q}_{p}^{d}$ denotes the vector space over $\mathbb{Q}_{p}$ which consists of all points x $=$ $\left(x_{1}, x_{2}, \ldots, x_{d}\right), x_{1}, x_{2}, \ldots, x_{d} \in \mathbb{Q}_{p}$. If we define $|\mathbf{x}|_{p}=\max _{1 \leq j \leq d}\left|x_{j}\right|_{p}$ for $\mathbf{x} \in \mathbb{Q}_{p}^{d}$, then it is easy to see that $|\cdot|_{p}$ is a non-Archimedean norm on $\mathbb{Q}_{p}^{d}$ and moreover $\mathbb{Q}_{p}^{d}$ is a locally compact Hausdorff and totally disconnected Banach space with respect to the norm $|\cdot|_{p}$. For $\gamma \in \mathbb{Z}$, we denote the ball $B_{\gamma}(\mathbf{a})$ with center $\mathbf{a} \in \mathbb{Q}_{p}^{d}$ and radius $p^{\gamma}$ and its boundary $S_{\gamma}(\mathbf{a})$ by

$$
\begin{aligned}
B_{\gamma}(\mathbf{a}) & =\left\{\mathbf{x} \in \mathbb{Q}_{p}^{d}:|\mathbf{x}-\mathbf{a}|_{p} \leq p^{\gamma}\right\}, \\
S_{\gamma}(\mathbf{a}) & =\left\{\mathbf{x} \in \mathbb{Q}_{p}^{d}:|\mathbf{x}-\mathbf{a}|_{p}=p^{\gamma}\right\},
\end{aligned}
$$

respectively. Since $\mathbb{Q}_{p}^{d}$ is a locally compact commutative group under addition, it follows from the standard analysis that there exists a unique Haar measure

Received May 21, 2009; Revised June 29, 2009.

2000 Mathematics Subject Classification. 11S80, 11K70, 11E95.

Key words and phrases. $p$-adic vector space, the $p$-adic Riesz and Bessel potential, Sobolev embedding theorem.

(C)2010 The Korean Mathematical Society 
$d_{H} \mathbf{x}$ on $\mathbb{Q}_{p}^{d}$ (up to positive constant multiple) which is translation invariant, i.e., $d_{H}(\mathbf{x}+\mathbf{a})=d_{H} \mathbf{x}$. We normalize the measure $d_{H} \mathbf{x}$ so that

$$
\int_{B_{0}(\mathbf{0})} d_{H} \mathbf{x} \fallingdotseq\left|B_{0}(\mathbf{0})\right|_{H}=1,
$$

where $|E|_{H}$ denotes the Haar measure of a measurable subset $E$ of $\mathbb{Q}_{p}^{d}$. From this integral theory, it is easy to obtain that $\left|B_{\gamma}(\mathbf{a})\right|_{H}=p^{\gamma d}$ and $\left|S_{\gamma}(\mathbf{a})\right|_{H}=$ $p^{\gamma d}\left(1-p^{-d}\right)$ for any $\mathbf{a} \in \mathbb{Q}_{p}^{d}$.

In what follows, we say that a complex-valued measurable function $f$ defined on $\mathbb{Q}_{p}^{d}$ is in $L^{q}\left(\mathbb{Q}_{p}^{d}\right), 1 \leq q \leq \infty$, if it satisfies

$$
\begin{aligned}
\|f\|_{L^{q}\left(\mathbb{Q}_{p}^{d}\right)} & \fallingdotseq\left(\int_{\mathbb{Q}_{p}^{d}}|f(\mathbf{x})|^{q} d_{H} \mathbf{x}\right)^{1 / q}<\infty, 1 \leq q<\infty, \\
\|f\|_{L^{\infty}\left(\mathbb{Q}_{p}^{d}\right)} & \left.\fallingdotseq \inf \left\{\alpha:\left|\left\{\mathbf{x} \in \mathbb{Q}_{p}^{d}:|f(\mathbf{x})|>\alpha\right\}\right|_{H}=0\right\}\right\}<\infty .
\end{aligned}
$$

Here the integral in (1.2) is defined as

$$
\begin{aligned}
\int_{\mathbb{Q}_{p}^{d}}|f(\mathbf{x})|^{q} d_{H} \mathbf{x} & =\lim _{N \rightarrow \infty} \int_{B_{N}(\mathbf{0})}|f(\mathbf{x})|^{q} d_{H} \mathbf{x} \\
& =\lim _{N \rightarrow \infty} \sum_{-\infty<\gamma \leq N} \int_{S_{\gamma}(\mathbf{0})}|f(\mathbf{x})|^{q} d_{H} \mathbf{x},
\end{aligned}
$$

if the limit exists. In general, the integral of a complex-valued measurable function $f$ on $\mathbb{Q}_{p}^{d}$ is defined as

$$
\int_{\mathbb{Q}_{p}^{d}} f(\mathbf{x}) d_{H} \mathbf{x}=\lim _{N \rightarrow \infty} \int_{B_{N}(\mathbf{0})} f(\mathbf{x}) d_{H} \mathbf{x}=\lim _{N \rightarrow \infty} \sum_{-\infty<\gamma \leq N} \int_{S_{\gamma}(\mathbf{0})} f(\mathbf{x}) d_{H} \mathbf{x},
$$

if the limit exists. We now mention some of the previous works on harmonic analysis on the $p$-adic field $\mathbb{Q}_{p}$ as follows; Haran $[2,3]$ obtained the explicit formula of Riesz potentials on $\mathbb{Q}_{p}$ and developed an analytical potential theory on the $p$-adic field $\mathbb{Q}_{p}$.

Let $f(\mathbf{x})$ be a complex-valued function on $\mathbb{Q}_{p}^{d}$. Then we say that $f$ is locallyconstant if for any $\mathbf{x} \in \mathbb{Q}_{p}^{d}$ there exists some integer $\ell(\mathbf{x}) \in \mathbb{Z}$ such that

$$
f\left(\mathbf{x}+\mathbf{x}^{\prime}\right)=f(\mathbf{x}),\left|\mathbf{x}^{\prime}\right|_{p} \leq p^{\ell(\mathbf{x})} .
$$

We denote by $\mathcal{E}\left(\mathbb{Q}_{p}^{d}\right)$ the class of all locally-constant functions on $\mathbb{Q}_{p}^{d}$ and we denote by $\mathcal{D}\left(\mathbb{Q}_{p}^{d}\right)$ the subclass of all functions in $\mathcal{E}\left(\mathbb{Q}_{p}^{d}\right)$ with compact support. We call a function in $\mathcal{D}\left(\mathbb{Q}_{p}^{d}\right)$ a test function on $\mathbb{Q}_{p}^{d}$. Since any nonzero $p$-adic number $x \in \mathbb{Q}_{p}$ with $|x|_{p}=p^{-\gamma}$ has the unique representation as in (1.1), we may define a function $\chi_{p}$ on $\mathbb{Q}_{p}$ by

$$
\chi_{p}(x)= \begin{cases}\prod_{j=\gamma}^{-1} \exp \left(2 \pi i x_{j} p^{j}\right), & \gamma<0, \\ 1, & \gamma \geq 0 \text { or } x=0 .\end{cases}
$$


Then it turns out (see [8]) that the function $\mathbf{x} \rightarrow \chi_{p}(\langle\boldsymbol{\xi}, \mathbf{x}\rangle)$ for each fixed $\boldsymbol{\xi} \in \mathbb{Q}_{p}^{d}$ is an additive character of the space $\mathbb{Q}_{p}^{d}$ and the group $B_{\gamma}(\mathbf{0})$, where $\langle\boldsymbol{\xi}, \mathbf{x}\rangle$ denotes the inner product of $\boldsymbol{\xi}, \mathbf{x} \in \mathbb{Q}_{p}^{d}$. For $g \in \mathcal{D}\left(\mathbb{Q}_{p}^{d}\right)$, we define the $p$-adic Fourier transformation $\mathfrak{F}[g]=\widetilde{g}$ of $g$ by

$$
\widetilde{g}(\boldsymbol{\xi})=\int_{\mathbb{Q}_{p}^{d}} \chi_{p}(\langle\boldsymbol{\xi}, \mathbf{x}\rangle) g(\mathbf{x}) d_{H} \mathbf{x}, \boldsymbol{\xi} \in \mathbb{Q}_{p}^{d} .
$$

Then $\mathfrak{F}$ is a unitary isomorphism from $\mathcal{D}\left(\mathbb{Q}_{p}^{d}\right)$ to $\mathcal{D}\left(\mathbb{Q}_{p}^{d}\right)$ with the inversion formula

$$
g(\mathbf{x})=\int_{\mathbb{Q}_{p}^{d}} \chi_{p}(-\langle\mathbf{x}, \boldsymbol{\xi}\rangle) \widetilde{g}(\boldsymbol{\xi}) d_{H} \boldsymbol{\xi}, g \in \mathcal{D}\left(\mathbb{Q}_{p}^{d}\right),
$$

and with the Parseval-Steklov equalities

$$
\begin{aligned}
& \int_{\mathbb{Q}_{p}^{d}} g(\mathbf{x}) \overline{h(\mathbf{x})} d_{H} \mathbf{x}=\int_{\mathbb{Q}_{p}^{d}} \widetilde{g}(\boldsymbol{\xi}) \overline{\widetilde{h}(\boldsymbol{\xi})} d_{H} \boldsymbol{\xi}, \\
& \int_{\mathbb{Q}_{p}^{d}} g(\mathbf{x}) \widetilde{h}(\mathbf{x}) d_{H} \mathbf{x}=\int_{\mathbb{Q}_{p}^{d}} \widetilde{g}(\boldsymbol{\xi}) h(\boldsymbol{\xi}) d_{H} \boldsymbol{\xi}, g, h \in \mathcal{D}\left(\mathbb{Q}_{p}^{d}\right) .
\end{aligned}
$$

Moreover, $\mathfrak{F}$ is a unitary isomorphism from $L^{2}\left(\mathbb{Q}_{p}^{d}\right)$ to $L^{2}\left(\mathbb{Q}_{p}^{d}\right)$ with the inversion formula

$$
g(\mathbf{x})=\lim _{\gamma \rightarrow \infty} \int_{B_{\gamma}(\mathbf{0})} \chi_{p}(-\langle\mathbf{x}, \boldsymbol{\xi}\rangle) \widetilde{g}(\boldsymbol{\xi}) d_{H} \boldsymbol{\xi} \text { in } L^{2}\left(\mathbb{Q}_{p}^{d}\right), \quad g \in \mathcal{D}\left(\mathbb{Q}_{p}^{d}\right),
$$

and with the Parseval-Steklov equalities on $L^{2}\left(\mathbb{Q}_{p}^{d}\right)$, because $\mathcal{D}\left(\mathbb{Q}_{p}^{d}\right)$ is a dense subset of $L^{2}\left(\mathbb{Q}_{p}^{d}\right)$ (see $\left.[8]\right)$.

Let $\mathcal{M}\left(\mathbb{Q}_{p}^{d}\right)$ denote the set of all complex-valued measurable functions on $\mathbb{Q}_{p}^{d}$. For $f, g \in \mathcal{M}\left(\mathbb{Q}_{p}^{d}\right)$, we define the convolution $f * g$ of $f$ and $g$ by

$$
f * g(\mathbf{x})=\int_{\mathbb{Q}_{p}^{d}} f(\mathbf{x}-\mathbf{y}) g(\mathbf{y}) d_{H} \mathbf{y}, \mathbf{x} \in \mathbb{Q}_{p}^{d} .
$$

For a complex number $z$ with $\operatorname{Re}(z)>0$, the $p$-adic Riesz kernel $\mathfrak{R}_{z}$ and Bessel kernel $\mathfrak{B}_{z}$ of order $z$ are defined by

$$
\mathfrak{R}_{z}(\mathbf{x})=\frac{1-p^{-z}}{1-p^{z-d}}|\mathbf{x}|_{p}^{-d+z}, \mathfrak{B}_{z}(\mathbf{x})=\mathfrak{F}^{-1}\left[\left(1+|\boldsymbol{\xi}|_{p}^{2}\right)^{-z / 2}\right](\mathbf{x}), \mathbf{x} \in \mathbb{Q}_{p}^{d},
$$

respectively; here, $\mathfrak{R}_{z}(\mathbf{x})$ and $\mathfrak{B}_{z}(\mathbf{x})$ are in fact multi-valued functions, and so we take their values only in the principle branch to guarantee the singlevaluedness. In what follows, we shall always assume that the values of every complex exponents are taken in the principle branch. Then we say that the operators $\mathcal{I}_{z}$ and $\mathcal{J}_{z}$ given by

$$
\mathcal{I}_{z}(f)(\mathbf{x})=\mathfrak{R}_{z} * f(\mathbf{x}), \mathcal{J}_{z}(f)(\mathbf{x})=\mathfrak{B}_{z} * f(\mathbf{x}), f \in \mathcal{M}\left(\mathbb{Q}_{p}^{d}\right),
$$

are the $p$-adic Riesz potential and Bessel potential of order $z$, respectively.

In what follows, we shall use notations; given two quantities $A$ and $B$, we write $A \lesssim B$ or $B \gtrsim A$ if there is a positive constant $c$ (possibly depending on 
the dimension $d$ and a prime number $p$ to be given) such that $A \leq c B$. We also write $A \sim B$ if $A \lesssim B$ and $B \lesssim A$. We denote by $\mathcal{C}_{F}$ the characteristic function of a measurable subset $F$ of $\mathbb{Q}_{p}^{d}$. For a complex number $z$, we write $z=s+i t$ where $s, t \in \mathbb{R}$.

Originally, Theorem 1.1 and Theorem 1.4 were obtained in [6]. In contrast to the method used in [6], we give a simple proof of them in this paper by using the $p$-adic version of the Calderón-Zygmund decomposition technique.

Theorem 1.1. Let $z$ be a complex number with $0<\operatorname{Re}(z)<d$ and let $1 \leq$ $q<r<\infty$ satisfy $1 / r=1 / q-\operatorname{Re}(z) / d$. If $q>1$, then there exists a constant $C_{0}=C(p, q, r, d)>0$ such that

$$
\left\|\mathcal{I}_{z}(f)\right\|_{L^{r}\left(\mathbb{Q}_{p}^{d}\right)} \leq C_{0}\|f\|_{L^{q}\left(\mathbb{Q}_{p}^{d}\right)}
$$

for any $f \in L^{q}\left(\mathbb{Q}_{p}^{d}\right)$. Moreover, $\mathcal{I}_{z}$ is of weak type $(1, r)$; that is to say, there is a constant $C_{1}=C_{1}(p, r, d)>0$ such that

$$
\left|\left\{\mathbf{x} \in \mathbb{Q}_{p}^{d}:\left|\mathcal{I}_{z}(f)(\mathbf{x})\right|>\lambda\right\}\right|_{H} \leq \frac{C_{1}}{\lambda^{r}}\|f\|_{L^{1}\left(\mathbb{Q}_{p}^{d}\right)}^{r}, \lambda>0
$$

for any $f \in L^{1}\left(\mathbb{Q}_{p}^{d}\right)$.

Corollary 1.2. Let $z$ be a complex number with $0<\operatorname{Re}(z)<d$ and let $1 \leq$ $q<r<\infty$ satisfy $1 / r=1 / q-\operatorname{Re}(z) / d$. If $q>1$, then there exists a constant $C_{2}=C_{2}(p, q, r, d)>0$ such that

$$
\left\|\mathcal{J}_{z}(f)\right\|_{L^{r}\left(\mathbb{Q}_{p}^{d}\right)} \leq C_{2}\|f\|_{L^{q}\left(\mathbb{Q}_{p}^{d}\right)}
$$

for any $f \in L^{q}\left(\mathbb{Q}_{p}^{d}\right)$. Moreover, $\mathcal{J}_{z}$ is of weak type $(1, r)$; that is to say, there is a constant $C_{3}=C_{3}(p, r, d)>0$ such that

$$
\left|\left\{\mathbf{x} \in \mathbb{Q}_{p}^{d}:\left|\mathcal{J}_{z}(f)(\mathbf{x})\right|>\lambda\right\}\right|_{H} \leq \frac{C_{3}}{\lambda^{r}}\|f\|_{L^{1}\left(\mathbb{Q}_{p}^{d}\right)}^{r}, \lambda>0
$$

for any $f \in L^{1}\left(\mathbb{Q}_{p}^{d}\right)$.

For $0 \leq s<d$ and $f \in L_{l o c}^{1}\left(\mathbb{Q}_{p}^{d}\right)$, we define the fractional maximal function $\mathcal{M}_{s}(f)$ by

$$
\mathcal{M}_{s}(f)(\mathbf{x})=\sup _{\gamma \in \mathbb{Z}} \frac{1}{p^{\gamma(d-s)}} \int_{B_{\gamma}(\mathbf{x})}|f(\mathbf{y})| d_{H} \mathbf{y}, \mathbf{x} \in \mathbb{Q}_{p}^{d} .
$$

Corollary 1.3. Let $0<s<d$ and let $1 \leq q<r<\infty$ satisfy $1 / r=1 / q-s / d$. If $q>1$, then there exists a constant $c_{0}=c_{0}(p, q, r, d)>0$ such that

$$
\left\|\mathcal{M}_{s}(f)\right\|_{L^{r}\left(\mathbb{Q}_{p}^{d}\right)} \leq c_{0}\|f\|_{L^{q}\left(\mathbb{Q}_{p}^{d}\right)}
$$

for any $f \in L^{q}\left(\mathbb{Q}_{p}^{d}\right)$. Moreover, $\mathcal{M}_{s}$ is of weak type $(1, r)$; that is to say, there is a constant $c_{1}=c_{1}(p, r, d)>0$ such that

$$
\left|\left\{\mathbf{x} \in \mathbb{Q}_{p}^{d}:\left|\mathcal{M}_{s}(f)(\mathbf{x})\right|>\lambda\right\}\right|_{H} \leq \frac{c_{1}}{\lambda^{r}}\|f\|_{L^{1}\left(\mathbb{Q}_{p}^{d}\right)}^{r}, \lambda>0
$$

for any $f \in L^{1}\left(\mathbb{Q}_{p}^{d}\right)$. 
For $s \geq 0$ and $1<q<\infty$, we denote by $L_{s}^{q}\left(\mathbb{Q}_{p}^{d}\right)$ the space of all generalized functions $u \in \mathcal{D}^{\prime}\left(\mathbb{Q}_{p}^{d}\right)$ such that $\mathfrak{F}^{-1}\left[\left(1+|\boldsymbol{\xi}|_{p}^{2}\right)^{s / 2} \widetilde{u}\right] \in L^{q}\left(\mathbb{Q}_{p}^{d}\right)$ and we call it the $p$-adic Sobolev space which has the norm $\|u\|_{L_{s}^{q}\left(\mathbb{Q}_{p}^{d}\right)}=\| \mathfrak{F}^{-1}[(1+$ $\left.\left.|\boldsymbol{\xi}|_{p}^{2}\right)^{s / 2} \widetilde{u}\right] \|_{L^{q}\left(\mathbb{Q}_{p}^{d}\right)}$. Then we obtain the $p$-adic analogue of the Sobolev embedding theorem.

Theorem 1.4. (a) Let $1<q \leq r<\infty$. If $s \geq 0$ is a real number satisfying $1 / q-1 / r=s / d$, then the space $L_{s}^{q}\left(\mathbb{Q}_{p}^{d}\right)$ is continuously embedded into $L^{r}\left(\mathbb{Q}_{p}^{d}\right)$.

(b) If $s>d / q$ and $q>1$, then the space $L_{s}^{q}\left(\mathbb{Q}_{p}^{d}\right)$ is continuously embedded into $L^{\infty}\left(\mathbb{Q}_{p}^{d}\right)$ and any element $f \in L_{s}^{q}\left(\mathbb{Q}_{p}^{d}\right)$ can be modified on a set $E \subset \mathbb{Q}_{p}^{d}$ with $|E|_{H}=0$ so that the resulting function is uniformly continuous.

\section{Preliminary estimates and the proof of main theorems}

In this section, we furnish several useful propositions and lemma and prove the main theorems.

Proposition 2.1. If $\mathfrak{m}$ is a complex-valued function on $\mathbb{R}_{+}$with

$$
\sum_{\gamma=0}^{\infty}\left|\mathfrak{m}\left(p^{-\gamma}\right)\right| p^{-\gamma d}<\infty
$$

then we have that for any $\mathbf{x} \in \mathbb{Q}_{p}^{d} \backslash\{\mathbf{0}\}$,

$$
\int_{\mathbb{Q}_{p}^{d}} \chi_{p}(-\langle\mathbf{x}, \boldsymbol{\xi}\rangle) \mathfrak{m}\left(|\boldsymbol{\xi}|_{p}\right) d_{H} \boldsymbol{\xi}=\frac{1-p^{-d}}{|\mathbf{x}|_{p}^{d}} \sum_{\gamma=0}^{\infty} p^{-\gamma d} \mathfrak{m}\left(p^{-\gamma}|\mathbf{x}|_{p}^{-1}\right)-\frac{1}{|\mathbf{x}|_{p}^{d}} \mathfrak{m}\left(p|\mathbf{x}|_{p}^{-1}\right)
$$

Proof. It easily follows from (1.4) and the change of variable that

$$
\int_{B_{\gamma}(\mathbf{0})} \chi_{p}(-\langle\mathbf{x}, \boldsymbol{\xi}\rangle) d_{H} \boldsymbol{\xi}=p^{\gamma d} \mathcal{C}_{B_{-\gamma}(\mathbf{0})}(\mathbf{x})
$$

for any $\gamma \in \mathbb{Z}$. Thus for $\gamma \in \mathbb{Z}$ we have that

$$
\begin{aligned}
\int_{S_{\gamma}(\mathbf{0})} \chi_{p}(-\langle\mathbf{x}, \boldsymbol{\xi}\rangle) d_{H} \boldsymbol{\xi} & =p^{\gamma d} \mathcal{C}_{B_{-\gamma}(\mathbf{0})}(\mathbf{x})-p^{(\gamma-1) d} \mathcal{C}_{B_{-\gamma+1}(\mathbf{0})}(\mathbf{x}) \\
& =p^{\gamma d}\left(1-p^{-d}\right) \mathcal{C}_{B_{-\gamma}(\mathbf{0})}(\mathbf{x})-p^{(\gamma-1) d} \mathcal{C}_{S_{-\gamma+1}(\mathbf{0})}(\mathbf{x})
\end{aligned}
$$

Hence by (2.1) and simple calculation we obtain that

$$
\begin{aligned}
\int_{\mathbb{Q}_{p}^{d}} \chi_{p}(-\langle\mathbf{x}, \boldsymbol{\xi}\rangle) \mathfrak{m}\left(|\boldsymbol{\xi}|_{p}\right) d_{H} \boldsymbol{\xi} & =\lim _{N \rightarrow \infty} \sum_{\gamma=-\infty}^{N} \mathfrak{m}\left(p^{\gamma}\right) \int_{S_{\gamma}(\mathbf{0})} \chi_{p}(-\langle\boldsymbol{\xi}, \mathbf{x}\rangle) d_{H} \mathbf{x} \\
& =\frac{1-p^{-d}}{|\mathbf{x}|_{p}^{d}} \sum_{\gamma=0}^{\infty} p^{-\gamma d} \mathfrak{m}\left(p^{-\gamma}|\mathbf{x}|_{p}^{-1}\right)-\frac{1}{|\mathbf{x}|_{p}^{d}} \mathfrak{m}\left(p|\mathbf{x}|_{p}^{-1}\right)
\end{aligned}
$$

Therefore we complete the proof. 
Lemma 2.2. If $z$ is a complex number with $0<\operatorname{Re}(z)<d$, then we have;

(a) $\left|\mathcal{B}_{z}(\mathbf{x})\right| \leq \frac{\left|1-p^{z-d}\right|}{\left|1-p^{-z}\right|} \frac{p^{\operatorname{Re}(z)}-2 p^{\operatorname{Re}(z)-d}+1}{p^{\operatorname{Re}(z)}\left(1-p^{\operatorname{Re}(z)-d}\right)}\left|\mathcal{R}_{z}(\mathbf{x})\right|, \mathbf{x} \in \mathbb{Q}_{p}^{d} \backslash\{\mathbf{0}\}$.

(b) $\left|\mathcal{B}_{z}(\mathbf{x})\right| \leq \frac{p^{\operatorname{Re}(z)}-2 p^{\operatorname{Re}(z)-d}+1}{p^{\operatorname{Re}(z)}\left(1-p^{\operatorname{Re}(z)-d}\right)} \frac{1}{|\mathbf{x}|_{p}^{d-\operatorname{Re}(z)}} \mathcal{C}_{B_{0}(\mathbf{0})}(\mathbf{x})+\frac{2}{|\mathbf{x}|_{p}^{d}} \mathcal{C}_{\mathbb{Q}_{p}^{d} \backslash B_{0}(\mathbf{0})}(\mathbf{x})$.

Proof. (a) It easily follows from Proposition 2.1 that

$$
\begin{aligned}
\left|\mathcal{B}_{z}(\mathbf{x})\right| & \leq \frac{1-p^{-d}}{|\mathbf{x}|_{p}^{d}} \sum_{\gamma=0}^{\infty} \frac{p^{-\gamma d}}{\left(1+p^{-2 \gamma}|\mathbf{x}|_{p}^{-2}\right)^{\operatorname{Re}(z) / 2}}+\frac{1}{|\mathbf{x}|_{p}^{d}\left(1+p^{2}|\mathbf{x}|_{p}^{-2}\right)^{\operatorname{Re}(z) / 2}} \\
& \leq\left(1-p^{-d}\right) \sum_{\gamma=\log _{p}\left(|\mathbf{x}|_{p}\right)}^{\infty} p^{-\gamma(d-\operatorname{Re}(z))}+\frac{1}{p^{\operatorname{Re}(z)}} \frac{1}{|\mathbf{x}|_{p}^{d-\operatorname{Re}(z)}} \\
& =\frac{1-p^{-d}}{1-p^{\operatorname{Re}(z)-d}} \frac{1}{|\mathbf{x}|_{p}^{d-\operatorname{Re}(z)}}+\frac{1}{p^{\operatorname{Re}(z)}} \frac{1}{|\mathbf{x}|_{p}^{d-\operatorname{Re}(z)}} \\
& =\frac{\left|1-p^{z-d}\right|}{\left|1-p^{-z}\right|} \frac{p^{\operatorname{Re}(z)}-2 p^{\operatorname{Re}(z)-d}+1}{p^{\operatorname{Re}(z)}\left(1-p^{\operatorname{Re}(z)-d}\right)}\left|\mathcal{R}_{z}(\mathbf{x})\right| .
\end{aligned}
$$

(b) The second part follows from calculation similar to (a) in two cases $|\mathbf{x}|_{p} \leq 1$ or $|\mathbf{x}|_{p}>1$. Therefore we complete the proof.

Proposition 2.3. Let $\mathfrak{b} \in L^{1}\left(\mathbb{Q}_{p}^{d}\right)$ be supported in a p-adic ball $B$ and satisfy

$$
\int_{\mathbb{Q}_{p}^{d}} \mathfrak{b}(\mathbf{x}) d_{H} \mathbf{x}=0
$$

If $q$ is a real number with $q>0$, then we have that

$$
\int_{\mathbb{Q}_{p}^{d} \backslash B}\left|\mathcal{I}_{z}(\mathfrak{b})(\mathbf{x})\right|^{q} d_{H} \mathbf{x}=0 .
$$

Proof. Since $|\cdot|_{p}$ is a non-Archimedean norm on $\mathbb{Q}_{p}^{d}$ and $\mathfrak{b}$ is supported in $B$, $|\mathbf{x}-\mathbf{y}|_{p}=|\mathbf{x}|_{p}$ for $\mathbf{x} \in \mathbb{Q}_{p}^{d} \backslash B$ and $\mathbf{y} \in B$. By the cancellation property of $\mathfrak{b}$ we easily obtain that

$$
\left|\mathcal{I}_{z}(\mathfrak{b})(\mathbf{x})\right| \leq \frac{\left|1-p^{-z}\right|}{\left|1-p^{z-d}\right|} \int_{\mathbb{Q}_{p}^{d}}|| \mathbf{x}-\left.\mathbf{y}\right|_{p} ^{-d+z}-|\mathbf{x}|_{p}^{-d+z}|| \mathfrak{b}(\mathbf{y}) \mid d_{H} \mathbf{y}=0
$$

provided that $\mathbf{x} \in \mathbb{Q}_{p}^{d} \backslash B$. This implies the required result.

Lemma 2.4. Let $z$ be a complex number satisfying $0<\operatorname{Re}(z)<d$. If $1 \leq q<$ $d / \operatorname{Re}(z)$, then there exists a constant $C_{4}=C_{4}(d, p, q, z)>0$ such that

$$
\left\|\mathcal{I}_{z}(f)\right\|_{L^{\infty}\left(\mathbb{Q}_{p}^{d}\right)} \leq C_{4}\|f\|_{L^{q}\left(\mathbb{Q}_{p}^{d}\right)}^{q \operatorname{Re}(z) / d}\|f\|_{L^{\infty}\left(\mathbb{Q}_{p}^{d}\right)}^{1-q \operatorname{Re}(z) / d}
$$

for any $f \in L^{q}\left(\mathbb{Q}_{p}^{d}\right) \cap L^{\infty}\left(\mathbb{Q}_{p}^{d}\right)$. 
Proof. Take any $f \in L^{q}\left(\mathbb{Q}_{p}^{d}\right) \cap L^{\infty}\left(\mathbb{Q}_{p}^{d}\right)$. Then we observe that for any $\gamma_{0} \in \mathbb{Z}$,

$$
\begin{aligned}
& \left|\mathcal{I}_{z}(f)(\mathbf{x})\right| \leq\|f\|_{L^{\infty}\left(\mathbb{Q}_{p}^{d}\right)} \int_{B_{\gamma_{0}}(\mathbf{0})}|\mathbf{y}|_{p}^{\operatorname{Re}(z)-d} d_{H} \mathbf{y} \\
& +\|f\|_{L^{q}\left(\mathbb{Q}_{p}^{d}\right)}\left(\int_{\mathbb{Q}_{p}^{d} \backslash\left\{B_{\gamma_{0}}(\mathbf{0})\right\}}|\mathbf{y}|_{p}^{(\operatorname{Re}(z)-d) q^{\prime}} d_{H} \mathbf{y}\right)^{1 / q^{\prime}} \\
& =\|f\|_{L^{\infty}\left(\mathbb{Q}_{p}^{d}\right)}\left(1-p^{-d}\right) \sum_{-\infty<\gamma \leq \gamma_{0}} p^{\gamma(\operatorname{Re}(z)-d)} p^{\gamma d} \\
& +\|f\|_{L^{q}\left(\mathbb{Q}_{p}^{d}\right)}\left(\left(1-p^{-d}\right) \sum_{\gamma=\gamma_{0}+1}^{\infty} p^{\gamma(\operatorname{Re}(z)-d) q^{\prime}} p^{\gamma d}\right)^{1 / q^{\prime}} \\
& =\|f\|_{L^{\infty}\left(\mathbb{Q}_{p}^{d}\right)} \frac{1-p^{-d}}{1-p^{-\operatorname{Re}(z)}} p^{\operatorname{Re}(z) \gamma_{0}} \\
& +\|f\|_{L^{q}\left(\mathbb{Q}_{p}^{d}\right)} \frac{\left(1-p^{-d}\right) p^{d-(d-\operatorname{Re}(z)) q^{\prime}}}{1-p^{d-(d-\operatorname{Re}(z)) q^{\prime}}} p^{\left[d-(d-\operatorname{Re}(z)) q^{\prime}\right] \gamma_{0}} .
\end{aligned}
$$

We now choose some $\gamma_{0} \in \mathbb{Z}$ in $(2.2)$ so that

$$
p^{\gamma_{0}}=\left(\frac{\|f\|_{L^{q}\left(\mathbb{Q}_{p}^{d}\right)}}{\|f\|_{L^{\infty}\left(\mathbb{Q}_{p}^{d}\right)}}\right)^{q / d}\left(1-p^{-d}\right)^{-1 / d}\left(1-p^{-\operatorname{Re}(z)}\right)^{q / d}\left(\frac{p^{d-(d-\operatorname{Re}(z)) q^{\prime}}}{1-p^{d-(d-\operatorname{Re}(z)) q^{\prime}}}\right)^{(q-1) / d} .
$$

Then it follows from (2.2) and simple computation that

$$
\left\|\mathcal{I}_{z}(f)\right\|_{L^{\infty}\left(\mathbb{Q}_{p}^{d}\right)} \leq C_{4}\|f\|_{L^{q}\left(\mathbb{Q}_{p}^{d}\right)}^{q \operatorname{Re}(z) / d}\|f\|_{L^{\infty}\left(\mathbb{Q}_{p}^{d}\right)}^{1-q \operatorname{Re}(z) / d},
$$

where the constant $C_{4}$ is explicitly given by

$$
C_{4}=2\left(1-p^{-d}\right)^{1-\operatorname{Re}(z) / d}\left(1-p^{-\operatorname{Re}(z)}\right)^{-1+q \operatorname{Re}(z) / d}\left(\frac{p^{d-(d-\operatorname{Re}(z)) q^{\prime}}}{1-p^{d-(d-\operatorname{Re}(z)) q^{\prime}}}\right)^{\frac{(q-1) \operatorname{Re}(z)}{d}} .
$$

Hence we complete the proof.

Proof of Theorem 1.1. First, we prove that the Riesz potential $\mathcal{I}_{z}$ is of weak type $(1, r)$. We observe that if $q=1$, then $r=d /(d-\operatorname{Re}(z))$. Take any $f \in L^{1}\left(\mathbb{Q}_{p}^{d}\right)$. For this estimate, we employ the $p$-adic version [4] of the CalderónZygmund decomposition of $f$ with aperture $\mu>0$ as follows;

$$
f=\mathfrak{g}+\mathfrak{b} \fallingdotseq \mathfrak{g}+\sum_{k=1}^{\infty} \mathfrak{b}_{k}
$$

where $\left\{B_{k}: k \in \mathbb{N}\right\}$ is a countable family of pairwise disjoint $p$-adic balls so that

(a) $\left|\left\{\mathbf{x} \in \mathbb{Q}_{p}^{d}:|\mathfrak{g}(\mathbf{x})|>p^{d} \mu\right\}\right|_{H}=0$,

(b) $\mathfrak{b}_{k}(\mathbf{x})=0$ for any $\mathbf{x} \in \mathbb{Q}_{p}^{d} \backslash B_{k}$ and $\int_{\mathbb{Q}_{p}^{d}} \mathfrak{b}_{k}(\mathbf{x}) d_{H} \mathbf{x}=0$,

(c) $\sum_{k=1}^{\infty}\left|B_{k}\right|_{H} \leq \frac{1}{\mu}\|f\|_{L^{1}\left(\mathbb{Q}_{p}^{d}\right)}$, 
(d) $\|\mathfrak{g}\|_{L^{1}\left(\mathbb{Q}_{p}^{d}\right)}+\sum_{k=1}^{\infty}\left\|\mathfrak{b}_{k}\right\|_{L^{1}\left(\mathbb{Q}_{p}^{d}\right)} \leq 3\|f\|_{L^{1}\left(\mathbb{Q}_{p}^{d}\right)}$.

We may assume that $\|f\|_{L^{1}\left(\mathbb{Q}_{p}^{d}\right)}=1$ by normalization. Applying Lemma 2.4 with $q=1$, we obtain that

$$
\begin{aligned}
\left\|\mathcal{I}_{z}(\mathfrak{g})\right\|_{L^{\infty}\left(\mathbb{Q}_{p}^{d}\right)} & \leq C_{4}\|f\|_{L^{1}\left(\mathbb{Q}_{p}^{d}\right)}^{\operatorname{Re}(z) / d}\|\mathfrak{g}\|_{L^{\infty}\left(\mathbb{Q}_{p}^{d}\right)}^{1-\operatorname{Re}(z) / d} \\
& \leq C_{4} 3^{\operatorname{Re}(z) / d} p^{d-\operatorname{Re}(z)} \mu^{1-\operatorname{Re}(z) / d} \\
& =C_{4} 3^{\operatorname{Re}(z) / d} p^{d-\operatorname{Re}(z)} \mu^{1 / r}
\end{aligned}
$$

If we set $\lambda=2 C_{4} 3^{\operatorname{Re}(z) / d} p^{d-\operatorname{Re}(z)} \mu^{1 / r} \fallingdotseq C_{1}^{1 / r} \mu^{1 / r}$, then we have that

$$
\left|\left\{\mathbf{x} \in \mathbb{Q}_{p}^{d}:\left|\mathcal{I}_{z}(f)(\mathbf{x})\right|>\lambda\right\}\right|_{H} \leq\left|\left\{\mathbf{x} \in \mathbb{Q}_{p}^{d}: \sum_{k \in \mathbb{N}}\left|\mathcal{I}_{z}\left(\mathfrak{b}_{k}\right)(\mathbf{x})\right|>\lambda / 2\right\}\right|_{H} .
$$

If we set $\Omega=\cup_{k \in \mathbb{N}} B_{k}$, then by (c) we get that $|\Omega|_{H} \leq 1 / \mu=C_{1} / \lambda^{r}$. It also follows from Proposition 2.3 that

$$
\begin{gathered}
\left|\left\{\mathbf{x} \in \mathbb{Q}_{p}^{d} \backslash \Omega: \sum_{k \in \mathbb{N}}\left|\mathcal{I}_{z}\left(\mathfrak{b}_{k}\right)(\mathbf{x})\right|>\lambda / 2\right\}\right|_{H}^{1 / r} \\
\leq \frac{2}{\lambda} \sum_{k \in \mathbb{N}}\left(\int_{\mathbb{Q}_{p}^{d} \backslash B_{k}}\left|\mathcal{I}_{z}\left(\mathfrak{b}_{k}\right)(\mathbf{x})\right|^{r} d_{H} \mathbf{x}\right)^{1 / r}=0 .
\end{gathered}
$$

Therefore we conclude that $\left|\left\{\mathbf{x} \in \mathbb{Q}_{p}^{d}:\left|\mathcal{I}_{z}(f)(\mathbf{x})\right|>\lambda\right\}\right|_{H} \leq C_{1} / \lambda^{r}$.

For $q>1$, we may assume that $\|f\|_{L^{q}\left(\mathbb{Q}_{p}^{d}\right)}=1$ by normalization. Then we have that

$$
\left\|\mathcal{I}_{z}(f)\right\|_{L^{r}\left(\mathbb{Q}_{p}^{d}\right)}^{r}=r \int_{0}^{\infty} \lambda^{r-1} \omega(\lambda) d \lambda,
$$

where $\omega(\lambda)=\left|\left\{\mathbf{x} \in \mathbb{Q}_{p}^{d}:\left|\mathcal{I}_{z}(f)(\mathbf{x})\right|>\lambda\right\}\right|_{H}$ for $\lambda>0$. In order to estimate $\omega(\lambda)$, we split $f$ into $f=g+h$ where $g=f \cdot \mathcal{C}_{\nu(\mu)}$ for $\nu(\mu)=\left\{\mathbf{x} \in \mathbb{Q}_{p}^{d}\right.$ : $|f(\mathbf{x})|>\mu\}$ and $\mu>0$. By Lemma 2.4, we obtain that

$$
\left\|\mathcal{I}_{z}(h)\right\|_{L^{\infty}\left(\mathbb{Q}_{p}^{d}\right)} \leq C_{4}\|h\|_{L^{q}\left(\mathbb{Q}_{p}^{d}\right)}^{q \operatorname{Re}(z) / d}\|h\|_{L^{\infty}\left(\mathbb{Q}_{p}^{d}\right)}^{1-q \operatorname{Re}(z) / d} \leq C_{4} \mu^{1-q \operatorname{Re}(z) / d}=C_{4} \mu^{q / r} .
$$

We now choose $\mu>0$ so that $\lambda / 2=C_{4} \mu^{q / r}$. From the weak type $\left(1, r_{0}\right)$ estimate of $\mathcal{I}_{z}$ with $r_{0}=d /(d-\operatorname{Re}(z))$ in the above, we see that

$$
\omega(\lambda) \leq\left|\left\{\mathbf{x} \in \mathbb{Q}_{p}^{d}:\left|\mathcal{I}_{z}(g)(\mathbf{x})\right|>\lambda / 2\right\}\right|_{H} \leq 2^{r_{0}} C_{1} \frac{\|g\|_{L^{1}\left(\mathbb{Q}_{p}^{d}\right)}^{r_{0}}}{\lambda^{r_{0}}} .
$$

Thus it follows from $(2.3),(2,4)$, and the $p$-adic versions of integral Minkowski's inequality and changing the order of integration that

$$
\begin{aligned}
\left\|\mathcal{I}_{z}(f)\right\|_{L^{r}\left(\mathbb{Q}_{p}^{d}\right)}^{r} & \leq r 2^{r_{0}} C_{1} \int_{0}^{\infty} \lambda^{r-1-r_{0}}\left(\int_{\nu(\mu)}|f(\mathbf{x})| d_{H} \mathbf{x}\right)^{r_{0}} d \lambda \\
& =q 2^{r} C_{1} C_{4}^{r-r_{0}} \int_{0}^{\infty} \mu^{q-1-q r_{0} / r}\left(\int_{\nu(\mu)}|f(\mathbf{x})| d_{H} \mathbf{x}\right)^{r_{0}} d \mu
\end{aligned}
$$




$$
\begin{aligned}
& \leq q 2^{r} C_{1} C_{4}^{r-r_{0}}\left(\int_{\mathbb{Q}_{p}^{d}}\left(\int_{0}^{|f(\mathbf{x})|} \mu^{q-1-q r_{0} / r} d \mu\right)^{1 / r_{0}}|f(\mathbf{x})| d_{H} \mathbf{x}\right)^{r_{0}} \\
& \leq q 2^{r} C_{1} C_{4}^{r-r_{0}}\left(\int_{\mathbb{Q}_{p}^{d}}|f(\mathbf{x})|^{q} d_{H} \mathbf{x}\right)^{r_{0}}=q 2^{r} C_{1} C_{4}^{r-r_{0}} .
\end{aligned}
$$

Therefore we complete the proof.

Proof of Corollary 1.2 and Corollary 1.3. It easily follows from Theorem 1.1, Lemma 2.2 , and the relation $\mathcal{M}_{s}(f) \lesssim \mathcal{I}_{s}(|f|)$ to be obtained by simple computation.

Proof of Theorem 1.4. (a) Let $f \in L_{s}^{q}\left(\mathbb{Q}_{p}^{d}\right)$ be given. For the proof, it suffices to prove that

$$
\|f\|_{L^{r}\left(\mathbb{Q}_{p}^{d}\right)} \leq C\left\|\mathfrak{F}^{-1}\left[\left(1+|\boldsymbol{\xi}|_{p}^{2}\right)^{s / 2} \widetilde{f}\right]\right\|_{L^{q}\left(\mathbb{Q}_{p}^{d}\right)} .
$$

If $s=0$, then we have $q=r$, and so the result is obvious. Thus we may assume that $s>0$. If we set $g(\mathbf{x})=\mathfrak{F}^{-1}\left[\left(1+|\boldsymbol{\xi}|_{p}^{2}\right)^{s / 2} \widetilde{f}\right](\mathbf{x})$, then proving $(2.5)$ is equivalent to showing the estimate

$$
\left\|\mathfrak{F}^{-1}\left[\left(1+|\boldsymbol{\xi}|_{p}^{2}\right)^{-s / 2} \widetilde{g}\right]\right\|_{L^{r}\left(\mathbb{Q}_{p}^{d}\right)} \leq C\|g\|_{L^{q}\left(\mathbb{Q}_{p}^{d}\right)} .
$$

We observe in $(2.6)$ that $\mathfrak{F}^{-1}\left[\left(1+|\boldsymbol{\xi}|_{p}^{2}\right)^{-s / 2} \widetilde{g}\right](\mathbf{x})=\mathfrak{B}_{s} * g(\mathbf{x})$. Since $1<q<$ $r<\infty$ and $1 / q-1 / r=s / d$ implies that $0<s<d$, the estimate (2.6) can immediately be obtained from Corollary 1.2.

(b) If $s>d / q$ and $q>1$, then we have $d-s<d(1-1 / q)$, and thus $(d-s) q^{\prime}<d$ where $q^{\prime}$ is the dual exponent of $q$. Then by (b) of Lemma 2.2 we have that $\mathfrak{B}_{s} \in L^{q^{\prime}}\left(\mathbb{Q}_{p}^{d}\right)$; indeed,

$$
\begin{aligned}
& \int_{\mathbb{Q}_{p}^{d}}\left|\mathfrak{B}_{s}(\mathbf{x})\right|^{q^{\prime}} d_{H} \mathbf{x} \\
\leq & \frac{p^{s}-2 p^{s-d}+1}{p^{s}\left(1-p^{s-d}\right)} \int_{B_{0}(\mathbf{0})} \frac{1}{|\mathbf{x}|_{p}^{(d-s) q^{\prime}}} d_{H} \mathbf{x}+2 \int_{\mathbb{Q}_{p}^{d} \backslash B_{0}(\mathbf{0})} \frac{1}{|\mathbf{x}|_{p}^{d q^{\prime}}} d_{H} \mathbf{x} \\
= & \frac{p^{s}-2 p^{s-d}+1}{p^{s}\left(1-p^{s-d}\right)}\left(1-p^{-d}\right) \sum_{\gamma=0}^{\infty} p^{-\gamma\left[d-(d-s) q^{\prime}\right]}+2\left(1-p^{-d}\right) \sum_{\gamma=1}^{\infty} p^{-\gamma d\left(q^{\prime}-1\right)} \\
= & \frac{p^{s}-2 p^{s-d}+1}{p^{s}\left(1-p^{s-d}\right)} \frac{1-p^{-d}}{1-p^{(d-s) q^{\prime}-d}}+\frac{2\left(1-p^{-d}\right) p^{d\left(1-q^{\prime}\right)}}{1-p^{d\left(1-q^{\prime}\right)}}<\infty .
\end{aligned}
$$

Let $f \in L_{s}^{q}\left(\mathbb{Q}_{p}^{d}\right)$ be given. For our proof, as in the above we have only to prove that

$$
\left\|\mathfrak{B}_{s} * g(\mathbf{x})\right\|_{L^{\infty}\left(\mathbb{Q}_{p}^{d}\right)} \leq C\|g\|_{L^{q}\left(\mathbb{Q}_{p}^{d}\right)},
$$

where $g(\mathbf{x})=\mathfrak{F}^{-1}\left[\left(1+|\boldsymbol{\xi}|_{p}^{2}\right)^{s / 2} \tilde{f}\right](\mathbf{x})$. Since $\mathfrak{B}_{s} \in L^{q^{\prime}}\left(\mathbb{Q}_{p}^{d}\right)$, the estimate $(2.7)$ can be obtained by applying the $p$-adic version of Hölder's inequality. 
If $\tau_{\mathbf{y}}$ denotes the translation operator defined by $\tau_{\mathbf{y}} f(\mathbf{x})=f(\mathbf{x}+\mathbf{y})$ for $\mathbf{y} \in \mathbb{Q}_{p}^{d}$, then it easily follows from $(2.7)$ that

$\left\|\tau_{\mathbf{y}} f-f\right\|_{L^{\infty}\left(\mathbb{Q}_{p}^{d}\right)}=\left\|\mathfrak{F}^{-1}\left[\left(1+|\boldsymbol{\xi}|_{p}^{2}\right)^{-s / 2}\left(\widetilde{\tau_{\mathbf{y}} g}-\widetilde{g}\right)\right]\right\|_{L^{\infty}\left(\mathbb{Q}_{p}^{d}\right)} \leq C\left\|\tau_{\mathbf{y}} g-g\right\|_{L^{q}\left(\mathbb{Q}_{p}^{d}\right)}$.

Thus we conclude that $\lim _{|\mathbf{y}|_{p} \rightarrow 0}\left\|\tau_{\mathbf{y}} f-f\right\|_{L^{\infty}\left(\mathbb{Q}_{p}^{d}\right)}=0$, because $g \in L^{q}\left(\mathbb{Q}_{p}^{d}\right)$. Hence this implies that $f$ can be modified on a set $E \subset \mathbb{Q}_{p}^{d}$ with $|E|_{H}=0$ so that the resulting function is continuous.

\section{References}

[1] Z. I. Borevich and I. R. Shafarevich, Number Theory, Academic press, New York, 1966

[2] S. Haran, Riesz potentials and explicit sums in arithmetic, Invent. Math. 101 (1990), 697-703.

[3] _ Analytic potential theory over the p-adics, Ann. Inst. Fourier(Grenoble) 43 (1993), no. 4, 905-944.

[4] Y.-C. Kim, Carleson measures and the BMO space on the p-adic vector space, Math. Nachr. 282 (2009), no. 9, 1278-1304.

[5] E. M. Stein, Harmonic Analysis; Real variable methods, orthogonality, and oscillatory integrals, Princeton Univ. Press, 1993.

[6] M. H. Taibleson, Fourier Analysis on Local Fields, Princeton Univ. Press, 1975.

[7] V. S. Vladimirov and I. V. Volovich, p-adic quantum mechanics, Commun. Math. Phys. 123 (1989), 659-676

[8] V. S. Vladimirov, I. V. Volovich, and E. I. Zelenov, p-adic Analysis and mathematical physics, Series on Soviet \& East European Mathematics, Vol. I, World Scientific, Singapore, 1992 .

Department of Mathematics Education

KOREA UNIVERSITY

SEOUl 136-701, KoreA

E-mail address: ychkim@korea.ac.kr 